\title{
A comprehensive study of the SX Phoenicis star BL Camelopardalis
}

\author{
S. Fauvaud ${ }^{1}$, E. Rodríguez ${ }^{2}$, A. Y. Zhou ${ }^{3,4}$, J. P. Sareyan ${ }^{5}$, I. Ribas ${ }^{6,7}$, M. D. Reed ${ }^{4}$, G. Klingenberg ${ }^{8}$, J. Farrell ${ }^{9}$, \\ J. Michelet ${ }^{10}$, S. E. Robinson ${ }^{11}$, G. Santacana ${ }^{1}$, and J. J. Rives ${ }^{1}$ \\ 1 Association AstroQueyras, Le bois de Bardon, Taponnat, 16110 La Rochefoucauld, France \\ e-mail: stephane.fauvaud@wanadoo.fr \\ 2 Instituto de Astrofísica de Andalucía, CSIC, PO Box 3004, 18080 Granada, Spain \\ e-mail: eloy@iaa.es \\ 3 National Astronomical Observatories, Chinese Academy of Sciences, Beijing 100012, PR China \\ 4 Department of Physics, Astronomy, and Materials Science, Missouri State University, 901 S. National, Springfield, MO 65897, \\ USA \\ 5 Observatoire de la Côte d'Azur, BP 4229, 06304 Nice Cedex 4, France \\ ${ }^{6}$ Institut de Ciéncies de l'Espai, CSIC, Facultat de Ciéncies, Campus UAB, Torre C5-parell, $2^{a}$ planta, 08193 Bellaterra, Spain \\ 7 Institut d'Estudis Espacials de Catalunya (IEEC), C/Gran Capitá 2-4, 08034 Barcelona, Spain \\ 8 Norwegian Astronomical Society, Engeshaugen 15, 8616 Mo i Rana, Norway \\ 9 Sulphur Flats Observatory, 449 Sulphur Creek Road, Jemez Springs, NM 87025, USA \\ ${ }^{10}$ Club d'Astronomie Lyon Ampère, 37 rue Paul Cazeneuve, 69008 Lyon, France \\ 11 American Association of Variable Star Observers, 25 Birch Street, Cambridge, MA 02138, USA
}

Received 18 July 2005 / Accepted 5 January 2006

\section{ABSTRACT}

\begin{abstract}
Context. BL Cam is an extreme field multiperiodic short-period low-metallicity high-amplitude SX Phe-type variable where its probably complex pulsational content is subject of controversies.

Aims. A comprehensive study has been carried out to investigate the nature and pulsational properties of this object.

Methods. The analysis is based on new photometric data collected during the last few years at different sites, as well as on all available previously published data. Frequency analyses have been performed on a number of reliable data sets to analyse the pulsational content. In addition, the classical O-C method was used to study the behaviour of the main period.

Results. Our frequency analysis confirms some aspects on the multiperiodicity of BL Cam, previously found by other authors, showing a number of secondary modes close to the main frequency $f_{0}=25.5769 \mathrm{~cd}^{-1}$. The secondary modes present eventual amplitude variations. However, the main frequency exhibits no significant changes in its amplitude and seems to correspond to the fundamental mode of radial pulsation. Abrupt changes sometimes observed in the light curves could be due to spurious activity on or close to the star's photosphere. We confirm the existence of a secondary frequency at 31.6 or $32.6 \mathrm{~cd}^{-1}$, with variable amplitude, and with the possibility of both frequencies being intrinsic to the star. More than one hundred of new times of light maxima have been determined in the present work. These together with those available in the literature allow us a more discriminating analysis of the $\mathrm{O}-\mathrm{C}$ diagram. This shows that the observed variations of the main period can be described by two terms: (i) a secular increase of the main period at a rate of $\mathrm{d} P / P \mathrm{~d} t=117( \pm 3) \times 10^{-9} \mathrm{yr}^{-1}$; and (ii) a perturbation from a companion star in a rather eccentric orbit with a period of $10.5( \pm 0.2)$ yr causing a light time semi-amplitude of $148( \pm 12) \mathrm{s}$.
\end{abstract}

Key words. stars: variables: delta Sct - stars: individual: BL Cam - stars: oscillations - techniques: photometric

\section{Introduction}

BL Cam (=GD 428, $\alpha=3^{\mathrm{h}} 47^{\mathrm{m}} 19^{\mathrm{s}}, \delta=+63^{\circ} 22^{\prime} 08^{\prime \prime}, \mathrm{J} 2000.0$, $\left.\langle V\rangle=13{ }^{\mathrm{m}} 1, \Delta V=0.33\right)$ is classified among the dozen field SX Phe-type pulsators known to date (Rodríguez \& Breger 2001). SX Phe stars are detected among field stars, globular clusters and nearby dwarf galaxies (Rodríguez \& López-González 2000). Most display visual peak-to-peak amplitudes larger than $\sim 0.3$ with short periods $(\leqslant 0.08)$ and some belong to double or multiple systems. SX Phe stars have typical Population II characteristics, i.e. they belong to the halo and old disk populations of the Galaxy with low metallicities and high spatial motions. However, they show surprisingly large masses $\left(\sim 1.0-1.2 M_{\odot}\right)$ and relatively young ages ( $\sim 2-5$ Gyr) (see e.g., Nemec \& Mateo 1990), characteristics which are still not fully explained by stellar evolution theory. The field SX Phe stars are pulsating variables located in the Lower Instability Strip, mixed with
Population I $\delta$ Sct-type variables. Recent reviews for the highamplitude members of this class can be found in Rodríguez (2003, 2004)

The discovery in the 1980's of three SX Phe variables in the globular cluster $\omega$ Cen (Rodríguez \& López-González 2000) confirmed that SX Phe and Population I $\delta$ Sct variables are different. At present, we know that the majority of the SX Phe variables located in globular clusters belong to their blue straggler (BS) population. These stars are bluer and brighter than the main sequence turn-off of the cluster (e.g., Mateo 1993) and their origin is still unclear with various hypotheses having been proposed in the literature: internal mixing in the atmosphere of single stars or mergers of two stars via mass transfer or direct collisions (Livio 1993; Stryker 1993). The connection between SX Phe stars and the BS phenomenon has been analysed by several authors (e.g. Eggen \& Iben 1989; Nemec \& Mateo 1990). Indeed, the investigation of the SX Phe-type variability can 
provide valuable advances in understanding the BS phenomenon and the history of the globular clusters themselves.

Additionally, as field high-amplitude SX Phe and $\delta$ Sct stars are known to be mostly monoperiodic or double-mode pulsators, their simple frequency spectra allow reliable mode identifications allowing the opportunity to use these pulsators to check the evolutionary tracks predicted by stellar evolution models (e.g., Høg \& Petersen 1997). Period-luminosity relations (Nemec et al. 1994; McNamara 1995) provide new distance scales independent of those derived from other methods, e.g., classical Cepheids or RR Lyr stars. This is of particular interest in globular clusters when a significant number of SX Phe variables are detected in the same cluster allowing precise periodluminosity relations to be determined and used as distance indicators (e.g., Pych et al. 2001; Mazur et al. 2003; Jeon et al. 2003, 2004).

BL Cam presents the second shortest period $(P=0.0391)$ among the known field SX Phe variables (the shortest one corresponds to PL43 = CS22966-043 (Preston \& Landolt 1998) with $P=0$.0374) and the shortest one among the high-amplitude objects of this type. BL Cam was originally reported by Giclas et al. (1970) as a possible white dwarf star. The variability of this object was discovered during a series of observations of white dwarf stars by Berg \& Duthie (1977). These authors also noted a recurrence of the light curve during 6 cycles over 5 days, a configuration that later turned out to be more complex than suggested by those preliminary results. An investigation of the basic parameters of BL Cam was carried out by McNamara \& Feltz (1978). In particular, their spectroscopic observations showed "an extreme weakness of the $\mathrm{K}$ line and the absence of other metal lines". The extreme metal-poor nature of this star $([\mathrm{Fe} / \mathrm{H}]=-2.4)$ was later confirmed by McNamara (1997).

Besides the main pulsation, a secondary periodicity was first identified by Hintz et al. (1997). Their photometric data suggested a radial double-mode pulsation nature with a primary $\left(f_{0}\right)$ to secondary $\left(f_{1}=32.6443 \mathrm{~cd}^{-1}\right)$ frequency ratio of $f_{0} / f_{1}=0.783$. This ratio fits the observed period-ratio versus metallicity relation established for the known fundamental/firstovertone double-mode high-amplitude radial pulsators in the Lower Instability Strip. However, different values for $f_{1}$ were determined by different authors using subsequent observations $\left(f_{1}=31.5912 \mathrm{~cd}^{-1}\right.$ by Zhou et al. (1999) and $f_{1}=32.3182 \mathrm{~cd}^{-1}$ by Kim et al. (2003) which lead to frequency ratios of $f_{0} / f_{1}=$ 0.810 and 0.791 , respectively). The reason of such different values for $f_{1}$ is still unknown and may be due to observational errors or to true changes in the pulsational content of BL Cam. Hence, it is important to solve this question as different frequency ratios have consequences in interpreting its double-mode nature. Furthermore, the data set collected by Zhou et al. (1999) revealed some additional modes of non-radial nature close to the main frequency $f_{0}$. If confirmed by new observations, BL Cam would have the most complex pulsation spectra among the field highamplitude SX Phe-type variables, and one of the most complex among the general population of SX Phe variables.

Hintz et al. (1997) were the first to detect a secular increase in BL Cam's primary frequency using the classical O-C method. A more detailed analysis of their O-C diagram, by Breger \& Pamyatnykh (1998), revealed a $0.02 \mathrm{~s}$ increase over the last 20 years. This gives $\mathrm{d} P / P \mathrm{~d} t=290 \times 10^{-9} \mathrm{y}^{-1}$, which is the largest period change detected in any high-amplitude $\delta$ Sct or SX Phe variable and cannot be explained by stellar evolutionary models. Nevertheless, Kim et al. (2003) pointed out a reversed trend of the O-C diagram since 1999 , leading to a possible sinusoidal behaviour, suggesting that BL Cam might be part of a binary system (with an orbital period of $\sim 25 \mathrm{yr}$, Rodríguez 2003). These controversies make the study of BL Cam even more compelling.

In this paper, new photometric observations of BL Cam have been carried out in order to perform a comprehensive study the behaviour of the main period, making use of the classical O-C method, as well as investigating the nature and pulsation properties of this extreme object.

\section{Observations}

The new photometric observations of BL Cam presented in this work were carried out at six observatories in 1996 and from 2003 to 2005. The summary and journal of new observations are given in Table 1. All observations were obtained with CCDs using various filters, including some observations without any filter.

Most of the data have been obtained with the $0.62 \mathrm{~m}$ telescope at the Château-Renard Observatory (2930 m altitude, Saint-Véran, French Alps), a facility operated by the ParisMeudon Observatory and AstroQueyras, an amateur association, and also with the $0.85 \mathrm{~m}$ telescope at the Xinglong Station, Beijing Astronomical Observatory (China). Some additional observations have been collected at Mo i Rana Observatory (Norway) with a $0.20 \mathrm{~m}$ telescope, at McDonald Observatory (USA) using the $2.10 \mathrm{~m}$ Otto Struve telescope, and at Sulphur Flats and Rockville observatories (USA) with 0.40 and $0.45 \mathrm{~m}$ telescopes, respectively. In total, more than $100 \mathrm{~h}$ of useful data were obtained on 28 nights.

\section{Data reduction}

Each CCD frame has been corrected for dark, bias and flatfield. All light measurements were made by synthetic aperture photometry. Atmospheric and differential colour extinction were also calculated and corrected. The typical photometric uncertainties (standard deviation) estimated from the differences between comparison stars are about 0.01 to 0 . 03 . The comparison stars have not shown any variability at this precision level during our observing runs.

Apart from their variable amplitude, possibly due to multiple periodic modulation (Zhou et al. 1999, and this work), the light curves of this variable star are strongly asymmetric, with sharp light maxima, and rather round-shaped light minima, occuring at phase $0.58( \pm 0.04)$ after the maxima. Figure 1 shows an example of a single night's light-curve of BL Cam.

The light curves of BL Cam can follow uncommon behaviours, with very abrupt decreases/increases of the apparent amplitudes from one cycle to another. For example, our October 2003 and February 2004 observations show that the amplitudes can vary widely ( $25 \%$ at HJD 2452925.5 or 2453053.3 , or $\sim 35 \%$ at HJD 2453051.3 ) on close successive cycles.

Our $B$ and $R$ observations obtained in 1996 at Château-Renard Observatory show that the colour index $(B-R)$ has a total amplitude of about 0.15 , the colour index mimima lag at phase $\sim 0.43$ after the maxima, i.e. significantly before phase 0.58 mentioned above for the light minima. The light curve decrease is quicker in blue than in red colour (or viceversa, the blue light increase starts before the red light increase), i.e. the light minima in $R$ filter occurs later than in $B$.

In all cases the data were used to determine new times of (the sharper) light maximum, by fitting a third degree polynomial on each observed peak. As such, we included 105 new timings of light maximum $\left(T_{\max }\right)$ in our ephemeris. The typical error on the 
Table 1. Journal of new observations. Observatory code: $\mathrm{ChR}=$ Château-Renard Observatory (France), $\mathrm{XL}=\mathrm{Xinglong}$ Station $(\mathrm{China}), \mathrm{SFO}=$ Sulphur Flats Observatory (USA), RO = Rockville Observatory (USA), McD = McDonald Observatory (USA), MR = Mo i Rana Observatory (Norway). Notes: a) 5 nights in filter $B$ ( $5 \mathrm{~h}$ of data and 7 times of maximum) and 4 nights in $R$ ( $4 \mathrm{~h}$ and 4 maxima), b) no filter was used on 2 of the 5 nights, c) filter $S$ is Schott BG40, centred at $\lambda=5000 \AA$ (Zhou et al. 2005), d) one night without filter and one night using Johnson $V$ filter.

\begin{tabular}{lccccrrr}
\hline \hline Date & Observatory & $\begin{array}{c}\text { Telescope } \\
(\mathrm{m})\end{array}$ & Filter & Nights & Hours & $T_{\max }$ & Notes \\
\hline 1996, Aug. & ChR & 0.62 & $B, R$ & 5 & 5 & 7 & $\mathrm{a}$ \\
2003, Sep. & ChR & 0.20 & no & 1 & 2 & 2 & \\
2003, Oct. & ChR & 0.62 & $R$ & 3 & 14 & 12 & \\
2003, Nov.-Dec. & XL & 0.85 & $V$ & 5 & 36 & 36 & $\mathrm{~b}$ \\
2003, Dec. & SFO & 0.40 & no & 1 & 3 & 3 & \\
2004, Feb. & ChR & 0.62 & $R$ & 4 & 16 & 16 & \\
2004, Feb. & RO & 0.45 & $V$ & 1 & 0.5 & 1 & \\
2004, Mar. & SFO & 0.40 & no & 1 & 3 & 3 & \\
2004, Sep. & McD & 2.10 & $S$ & 3 & 3 & 2 & $\mathrm{c}$ \\
2004, Oct. & MR & 0.20 & $V$ & 1 & 11 & 11 & \\
2005, Jan.-Feb. & MR & 0.20 & $V$ & 2 & 7 & 8 & $\mathrm{~d}$ \\
2005, Apr. & MR & 0.20 & no & 1 & 4 & 4 & \\
\hline
\end{tabular}

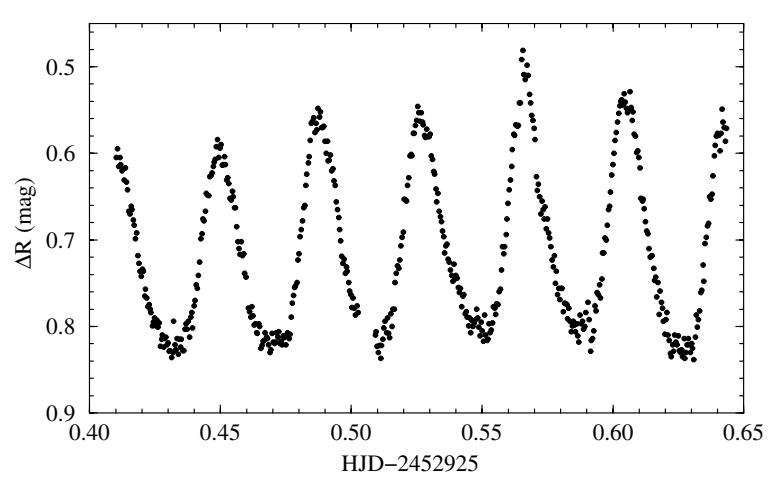

Fig. 1. $R$ light curve of BL Cam observed on October 13, 2003 at Château-Renard Observatory. $\Delta R$ means magnitude differences variable minus comparison star.

time of the individual maxima, is estimated at around 0.0004 , i.e. less than $1 \%$ of the main period of this variable. Table 2 displays the new observed times of light maximum.

\section{Frequency analysis}

Frequency analysis was carried out on each of the new data sets presented in this work and on those available in the literature that we considered as reliable enough for this purpose. The followed procedure is described in Rodríguez et al. (1998), where single-frequency and multiple-frequency techniques are combined using both Fourier and multiple least squares algorithms. The multiple-frequency least squares technique always calculates the best fit with all frequencies considered simultaneously.

The purpose of this analysis is to study the multiperiodic content of the light curves in order (i) to confirm the existence of the secondary frequency around $31-32 \mathrm{~cd}^{-1}$ detected by other authors, and determine its real value; (ii) to investigate the existence of eventual additional frequencies detected by Zhou et al. (1999); (iii) to study the multiperiodic behaviour over time (i.e. the number of observed frequencies and possible amplitude variations); and (iv) to investigate the behaviour of the main frequency $f_{0}$ with time (i.e. its possible amplitude variations).

In our analysis, we assume that the value of the main frequency is already known as $f_{0}=25.5769 \mathrm{~cd}^{-1}$, which corresponds to the period $P_{0}=0.03909785$ derived by
Zhou et al. (1999, Eq. (2)). The mentioned strong asymmetry in the shape of the light curve obviously generates the harmonics $2 f_{0}$ and $3 f_{0}$ in the Fourier analysis, their amplitudes and phases giving a mathematical representation of the asymmetry itself, an asymmetry common to most large amplitude variables (i.e. undergoing nonlinear physical processes). In order to make our analysis as homogeneous as possible, in each data set we first extracted the frequencies $f_{0}, 2 f_{0}$ and $3 f_{0}$ and then analysed the residuals. A peak of frequency has been considered as significant as soon as it fulfils the criterion given by Breger et al. $(1993,1996)$, i.e. an amplitude signal/noise $(S / N)$ ratio $\geq 4.0$ for independent peaks and 3.5 for peaks corresponding to frequency combinations (Breger et al. 1999).

In order to compare amplitudes obtained in different filters, all of them have been converted to equivalent amplitudes in the Johnson's $V$ filter. When observations were collected in $B$ or $R$ filters, the corresponding amplitudes can be transformed to $V$ equivalent amplitudes following Balona \& Evers (1999, Fig. 2), assuming a typical $\delta$ Sct regime. The following transformation equations were determined: $\Delta V=1.36( \pm 0.04) \Delta R$ and $\Delta V=0.80( \pm 0.02) \Delta B$. Data collected at McDonald Observatory in 2004 were obtained in filter S (i.e. Schott BG 40), which has a peak transmission around $\lambda=5000 \AA$ (Zhou et al. 2005). The corresponding equivalent $V$ amplitudes were obtained following the procedure described in Rodríguez (1999), which yields $\Delta V=0.88( \pm 0.03) \Delta S$.

\subsection{Results}

\subsubsection{Amplitude variations of the main frequency}

We first investigated the possibility of amplitude changes in the main frequency $f_{0}$ on the time scale of years. Table 3 lists the amplitudes of $f_{0}$ along with the secondary frequency $f_{1}$, when detected in the range $31-32 \mathrm{~cd}^{-1}$. No significant changes are detected in the amplitude of $f_{0}$ which is stable at 146 mmag within $\sim 10$ mmag. It must be noted that the error bars listed in this table are the "formal" error bars as a result of the Fourier fitting, but the "true" ones are slightly larger. Also, some data sets are not long enough, which can induce scatter in the solution of the fits. The amplitudes of $f_{0}$ can also be disturbed by the existence of secondary peaks close to $f_{0}$ which are not obviously discerned in short data sets (see Sect. 4.1.3). 
Table 2. New times of light maximum of BL Cam. $T_{\max }$ is in HJD-2 400 000. Observatory code as in Table 1 . Whenever quoted $B, R$ it means that the corresponding $T_{\max }$ was determined as the mean value.

\begin{tabular}{|c|c|c|c|c|c|c|c|c|}
\hline$\overline{T_{\max }}$ & Filter & Obs. & $\overline{T_{\max }}$ & Filter & Obs. & $\overline{T_{\max }}$ & Filter & Obs. \\
\hline 50311.5587 & $\bar{B}$ & ChR & 52990.2350 & $\bar{V}$ & $\mathrm{XL}$ & 53052.4427 & $R$ & ChR \\
\hline 50311.5998 & $B$ & ChR & 52990.2747 & $V$ & XL & 53053.3008 & $R$ & ChR \\
\hline 50312.5756 & $B, R$ & ChR & 52990.3126 & $V$ & XL & 53053.3406 & $R$ & ChR \\
\hline 50313.5926 & $B, R$ & ChR & 52990.3906 & $V$ & XL & 53053.3808 & $R$ & ChR \\
\hline 50318.5554 & $B, R$ & ChR & 52990.9775 & V & XL & 53053.4190 & $R$ & ChR \\
\hline 50318.5957 & $B$ & ChR & 52991.0172 & V & XL & 53082.6236 & no & SFO \\
\hline 50319.5338 & $B, R$ & ChR & 52991.0564 & V & XL & 53082.6633 & no & SFO \\
\hline 52906.5654 & no & ChR & 52991.0945 & $V$ & XL & 53082.7033 & no & SFO \\
\hline 52906.6442 & no & ChR & 52991.1338 & $V$ & XL & 53264.9755 & $S$ & $\mathrm{McD}$ \\
\hline 52925.2948 & $R$ & ChR & 52991.1734 & $V$ & XL & 53265.9535 & $S$ & $\mathrm{McD}$ \\
\hline 52925.3330 & $R$ & ChR & 52991.9542 & $V$ & XL & 53293.2440 & V & MR \\
\hline 52925.4518 & $R$ & ChR & 52991.9941 & V & XL & 53293.2830 & V & MR \\
\hline 52925.4900 & $R$ & ChR & 52992.0332 & $V$ & $\mathrm{XL}$ & 53293.3213 & V & MR \\
\hline 52925.5290 & $R$ & ChR & 52992.0717 & $V$ & XL & 53293.3610 & V & MR \\
\hline 52925.5693 & $R$ & ChR & 52992.1112 & $V$ & XL & 53293.4006 & $V$ & MR \\
\hline 52925.6072 & $R$ & ChR & 52992.1516 & $V$ & XL & 53293.4389 & $V$ & MR \\
\hline 52927.3673 & $R$ & ChR & 52992.2278 & $V$ & XL & 53293.4783 & $V$ & MR \\
\hline 52928.3047 & $R$ & ChR & 52992.2682 & $V$ & XL & 53293.5176 & $V$ & MR \\
\hline 52928.3437 & $R$ & ChR & 52992.3063 & $V$ & XL & 53293.5566 & $V$ & MR \\
\hline 52928.4229 & $R$ & ChR & 52992.3463 & $V$ & XL & 53293.5961 & $V$ & MR \\
\hline 52928.4617 & $R$ & ChR & 52992.3848 & $V$ & XL & 53293.6739 & $V$ & MR \\
\hline 52952.2319 & $V$ & XL & 53002.5893 & no & SFO & 53395.1709 & no & MR \\
\hline 52952.2707 & V & XL & 53002.6296 & no & SFO & 53395.2116 & no & MR \\
\hline 52952.3095 & $V$ & XL & 53002.6687 & no & SFO & 53395.2504 & no & MR \\
\hline 52952.3487 & $V$ & XL & 53044.6202 & $V$ & RO & 53395.2888 & no & MR \\
\hline 52954.1870 & $V$ & XL & 53050.4470 & $R$ & ChR & 53424.3004 & $V$ & MR \\
\hline 52954.2260 & $V$ & XL & 53050.4866 & $R$ & ChR & 53424.3400 & $V$ & MR \\
\hline 52954.2653 & $V$ & XL & 53051.2702 & $R$ & ChR & 53424.3803 & $V$ & MR \\
\hline 52954.3041 & $V$ & XL & 53051.3072 & $R$ & ChR & 53424.4194 & V & MR \\
\hline 52954.3436 & V & XL & 53051.3477 & $R$ & ChR & 53478.3752 & no & MR \\
\hline 52954.3829 & V & XL & 53051.3858 & $R$ & ChR & 53478.4129 & no & MR \\
\hline 52990.0389 & $V$ & XL & 53051.4236 & $R$ & ChR & 53478.4540 & no & MR \\
\hline 52990.0781 & V & XL & 53052.2840 & $R$ & ChR & 53478.4921 & no & MR \\
\hline 52990.1182 & $V$ & XL & 53052.3245 & $R$ & ChR & & & \\
\hline 52990.1574 & $V$ & XL & 53052.3639 & $R$ & ChR & & & \\
\hline 52990.1962 & V & XL & 53052.4044 & $R$ & ChR & & & \\
\hline
\end{tabular}

\subsubsection{The $31-32 \mathrm{~cd}^{-1}$ range}

In exploring the multiperiodic nature of BL Cam, the first aim is to determine the existence and true value of the secondary peak $f_{1}$ claimed by some authors in the region $31-32 \mathrm{~cd}^{-1}$. Previously published results give values of $32.6443 \mathrm{~cd}^{-1}$ (Hintz et al. 1997), $31.5912 \mathrm{~cd}^{-1}$ (Zhou et al. 1999), $32.3182 \mathrm{~cd}^{-1}$ (Kim et al. 2003), and $32.517 \mathrm{~cd}^{-1}$ (Delaney et al. 2000, unpublished). These differing results for $f_{1}$ can be caused by too short or not reliable enough data sets (high noise levels as compared with the low amplitude commonly found for $f_{1}$ ).

The results in Table 3 confirm that $f_{1}$ reliably exists and that its value is close to $31.6 \mathrm{~cd}^{-1}$ or $32.6 \mathrm{~cd}^{-1}$. At least one of these frequencies is present in each data set, although not always detected, and with amplitudes that show a rather large dispersion.

A summary of the frequencies detected in the range $31-32 \mathrm{~cd}^{-1}$ is given in Table 4 . They cluster around the mean values $31.64( \pm 0.05)$ and $32.64( \pm 0.10) \mathrm{cd}^{-1}$. The difference between these two frequencies might be due to the well-known $1 \mathrm{~cd}^{-1}$ alias problem, inherent in single-site data. The frequencies around $32.6 \mathrm{~cd}^{-1}$ show a higher dispersion and, if we consider the two better data sets (i.e. those collected in 1996 and 1999 by Zhou et al. 1999, 2001), $f_{1}$ should be $31.6 \mathrm{~cd}^{-1}$.

Is $f_{1}$ really a one peak solution with a $1 \mathrm{~cd}^{-1}$ aliasing problem? When analysing the 1999 data set of Zhou et al. (2001), the best published photometric run for BL Cam, we find the frequency $31.6759 \mathrm{~cd}^{-1}$ along with another peak at $32.5456 \mathrm{~cd}^{-1}$ (Tables 4 and 6). This suggests that, at least in some data sets, the peaks detected around $31.6 \mathrm{~cd}^{-1}$ and those around $32.6 \mathrm{~cd}^{-1}$ are intrinsic rather than produced by the $1 \mathrm{~cd}^{-1}$ alias. Possible explanations could be that (i) in some data sets only one frequency is detected because the spectrum resolution is not sufficient to resolve the two frequencies; and/or (ii) amplitude changes lead to sometimes detecting one frequency rather than the other.

\subsubsection{Multiperiodicity and microvariability in the $25 \mathrm{~cd}^{-1}$ region}

In addition to $f_{0}$ and its two first harmonics $\left(2 f_{0}, 3 f_{0}\right)$ and $f_{1}$ (in the region $31-32 \mathrm{~cd}^{-1}$ ), some other peaks have been detected in the power spectra of BL Cam by Zhou et al. (1999), in the region close to the main frequency $f_{0}$. Some frequency combinations of the new peaks with $f_{0}$ were also found by the same authors. This suggests the existence of microvariability in this star, with additional peaks corresponding to nonradial modes. Sometimes these new peaks have amplitudes larger than $f_{1}$. Similar microvariability has been previously found in some other high-amplitude $\delta$ Sct and SX Phe-type variables (SX Phe itself and DY Peg by Garrido \& Rodríguez 1996; AI Vel by Walraven et al. 1992; RV Ari by Pócs et al. 2002; RY Lep by Rodríguez et al. 2003). 


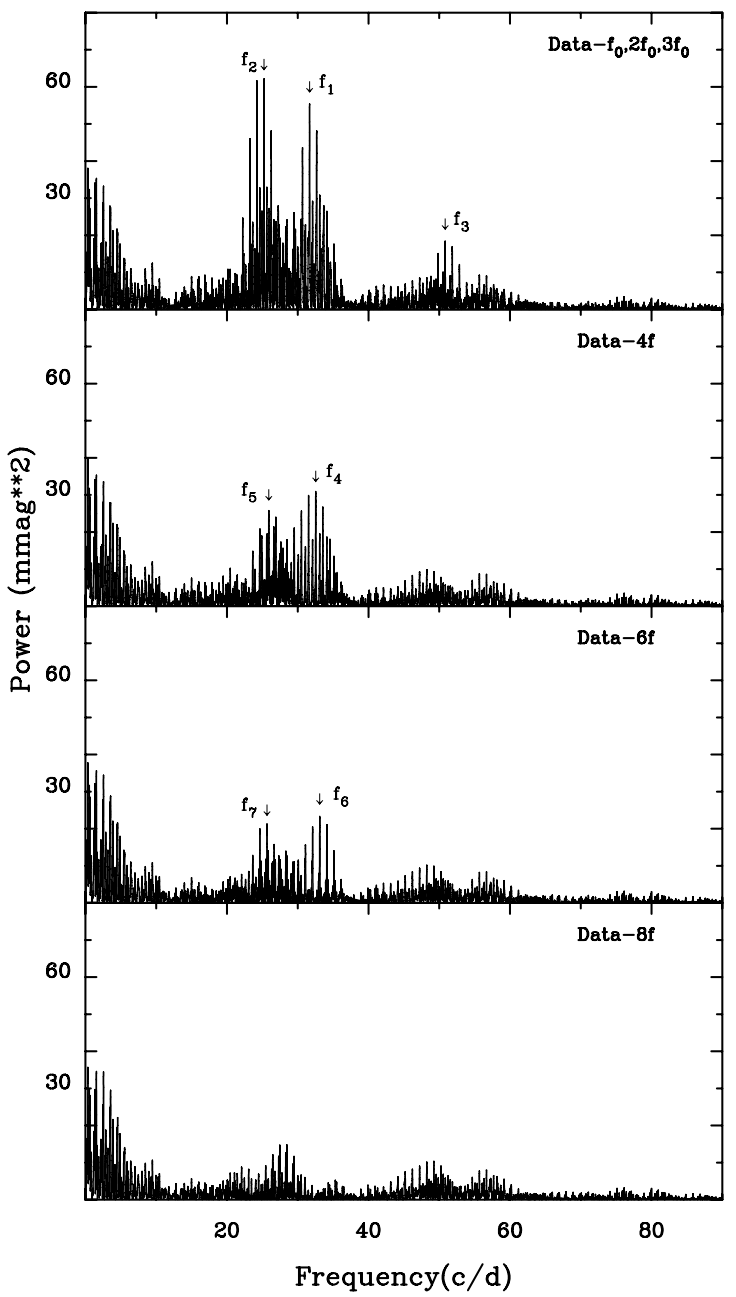

Fig. 2. Power spectra of BL Cam corresponding to the 1999 data set collected by Zhou et al. (2001). Panels: 1 ) residuals after removing $f_{0}$, $2 f_{0}$ and $\left.3 f_{0}, 2\right)$ same after removing $f_{0}-f_{3}, 3$ ) same after removing $f_{0}-f_{5}$ and 4 ) same after removing $f_{0}-f_{7}$.

In order to investigate microvariability in BL Cam, we have re-analysed the data sets available in the bibliography which are reliable for this task: those of Hintz et al. (1997), Kim et al. (2003), Wolf et al. (2002) and Zhou et al. (1999, 2001). In addition, our best observation runs were also investigated: those obtained at Xinglong Station (in the period November-December 2003) and Château-Renard Observatory (October 2003).

We first extracted the set of frequencies $f_{0}=25.5769 \mathrm{~cd}^{-1}$, $2 f_{0}$ and $3 f_{0}$ and then analysed the residuals, as described above. The new significant peaks detected have then been simultaneously optimized and removed from the data according to Rodríguez et al. (1998).

We will discuss in some detail the results obtained for the data sets of Zhou et al. (1999, 2001) which are listed in Tables 5 and 6 together with the corresponding $S / N$ values. For simplicity, the same names are assigned to the same peaks in both tables, whatever their amplitudes. Our results are in very good agreement with those published in Zhou et al. (1999). In addition to $f_{1}=31.5927 \mathrm{~cd}^{-1}$, other additional independent frequencies $f_{2}$, $f_{5}$ and $f_{7}$ are detected together with the combinations $f_{0}+f_{2} \sim f_{3}$ and $f_{0}+f_{5} \sim f_{8}$, and the amplitudes of $f_{2}$ and $f_{5}$ are larger than $f_{1}$. We do not detect at a significant level the linear combination $f_{0}+f_{1}$ reported by Zhou et al. (1999). On the other hand, a new significant peak at $f_{7}=25.6441 \mathrm{~cd}^{-1}$ and with $S / N=4.4$ is
Table 3. Amplitudes, $\Delta V_{0}$ and $\Delta V_{1}$, of $f_{0}=25.5769 \mathrm{~cd}^{-1}$ and $f_{1}$, respectively, determined from different data sets by Fourier analysis.

\begin{tabular}{|c|c|c|c|c|c|}
\hline Year & $\begin{array}{c}\Delta V_{0} \\
(\mathrm{mmag})\end{array}$ & $\begin{array}{c}\begin{array}{c}f_{1} \\
\left(\mathrm{~cd}^{-1}\right)\end{array} \\
\end{array}$ & $\begin{array}{c}\Delta V_{1} \\
(\mathrm{mmag})\end{array}$ & Source & Notes \\
\hline 1993 & $\begin{array}{r}140.4 \\
2.6\end{array}$ & - & - & 1 & \\
\hline 1994 & $\begin{array}{r}160.3 \\
2.1\end{array}$ & - & - & 1 & \\
\hline 1995 & $\begin{array}{r}146.5 \\
1.3\end{array}$ & 32.567 & $\begin{array}{l}9.0 \\
1.3\end{array}$ & 1 & \\
\hline 1996 & $\begin{array}{r}141.6 \\
2.0\end{array}$ & - & - & 1 & \\
\hline 1996 & $\begin{array}{r}142.8 \\
4.1\end{array}$ & 32.689 & $\begin{array}{r}13.2 \\
4.1\end{array}$ & 2 & $\mathrm{a}$ \\
\hline 1996 & $\begin{array}{r}159.8 \\
4.4\end{array}$ & 32.706 & $\begin{array}{r}13.7 \\
4.4\end{array}$ & 2 & b \\
\hline 1996 & $\begin{array}{r}146.8 \\
1.1\end{array}$ & 31.593 & $\begin{array}{r}11.5 \\
1.1\end{array}$ & 3 & \\
\hline 1999 & $\begin{array}{r}145.7 \\
0.5\end{array}$ & 31.676 & $\begin{array}{l}7.0 \\
0.5\end{array}$ & 4 & $\mathrm{c}$ \\
\hline 2000 & $\begin{array}{r}149.1 \\
1.3\end{array}$ & 31.683 & $\begin{array}{r}10.0 \\
1.3\end{array}$ & 5 & \\
\hline 2001 & $\begin{array}{r}151.9 \\
2.6\end{array}$ & - & - & 5 & \\
\hline 2001 & $\begin{array}{r}138.1 \\
1.3\end{array}$ & 31.629 & $\begin{array}{r}17.0 \\
1.3\end{array}$ & 6 & \\
\hline 2003 & $\begin{array}{r}153.8 \\
1.0\end{array}$ & 32.711 & $\begin{array}{r}14.4 \\
1.0\end{array}$ & 2 & d \\
\hline 2003 & $\begin{array}{r}146.9 \\
1.0\end{array}$ & 30.618 & $\begin{array}{l}9.6 \\
1.0\end{array}$ & 7 & e \\
\hline 2004 & $\begin{array}{r}137.3 \\
5.4\end{array}$ & - & - & 8 & $\mathrm{f}$ \\
\hline 2004 & $\begin{array}{r}144.8 \\
2.4\end{array}$ & - & - & 9 & \\
\hline 2005 & $\begin{array}{r}149.3 \\
5.8\end{array}$ & - & - & 9 & \\
\hline
\end{tabular}

Sources: 1) Hintz et al. (1997), 2) present work, Château-Renard Observatory, 3) Zhou et al. (1999), 4) Zhou et al. (2001), 5) Kim et al. (2003), 6) Wolf et al. (2002), 7) present work, Xinglong Station, 8) present work, McDonald Observatory, 9) present work, Mo i Rana Observatory. Notes: a) observations in Johnson $B$ filter, $\Delta V$ is determined from $\Delta V=0.80 \Delta B$ (see text), b) observations in $R$ filter, $\Delta V$ is determined from $\Delta V=1.36 \Delta R$ (see text), c) another peak is detected at $32.5456 \mathrm{~cd}^{-1}$, with $\Delta V=5.6( \pm 0.5) \mathrm{mmag}$ and $S / N=5.6$, d) this data set include the data from October 2003 to February 2004. $\Delta V$ is calculated from $\Delta V=1.36 \Delta R$ (see text), e) $f_{1}=30.618 \mathrm{~cd}^{-1}$ can be an $1 \mathrm{~cd}^{-1}$ alias of $31.6 \mathrm{~cd}^{-1}$, although the corresponding amplitude $S / N$ ratio is rather low $(S / N=3.9)$, f) observations in filter $S$ (Schott BG40), $\Delta V$ is determined from $\Delta V=0.88 \Delta S$ (see text).

detected in our analysis. This is very close to $f_{7}=25.6653 \mathrm{~cd}^{-1}$ that we find in our re-analysis of Zhou et al.'s (2001) data.

Table 6 and Fig. 2 show the results obtained for the data set collected in 1999 by Zhou et al. (2001). This is the best run available in the bibliography with about $50 \mathrm{~h}$ of observations and 2700 measurements collected during 17 nights spanning 67 days. The majority of these nights are of excellent photometric quality. The results of our analysis confirm the majority of the modes detected by Zhou et al. (1999). In particular, the independent modes $f_{2}=25.2469 \mathrm{~cd}^{-1}, f_{5}=25.9122 \mathrm{~cd}^{-1}$ and $f_{7}=25.6653 \mathrm{~cd}^{-1}$ are confirmed. However, their amplitudes are much smaller here and the combination $f_{0}+f_{5}$ is not detected; only the combination $f_{0}+f_{2}$ is significantly detected. Interestingly, in addition to the frequency $f_{1}=31.6759 \mathrm{~cd}^{-1}$, another peak is clearly detected at $f_{4}=32.5456 \mathrm{~cd}^{-1}$, as discussed in the previous section. 
Table 4. Summary of frequencies in the range $31-32 \mathrm{~cd}^{-1}$. (*) Possible alias (see Note e) in Table 3).

\begin{tabular}{cll}
\hline \hline Year & $\begin{array}{c}\sim 31.64 \\
\left(\mathrm{~cd}^{-1}\right)\end{array}$ & $\begin{array}{c}\sim 32.64 \\
\left(\mathrm{~cd}^{-1}\right)\end{array}$ \\
\hline 1995 & 31.593 & 32.567 \\
1996 & & 32.689 \\
& & 32.706 \\
1999 & 31.676 & 32.546 \\
2000 & 31.683 & 32.517 \\
2001 & 31.629 & \\
2003 & $30.618\left(^{*}\right)$ & 32.711 \\
& & \\
$\Delta V_{1}(\mathrm{mmag})$ & 7 to 17 & $\sim 6$ to 14 \\
\hline
\end{tabular}

Table 5. Results of Fourier analysis applied to the 1996 data set of Zhou et al. (1999). The sigma of the residuals is $28.6 \mathrm{mmag}$.

\begin{tabular}{lrr}
\hline \hline $\begin{array}{l}\text { Frequency } \\
\left(\mathrm{cd}^{-1}\right)\end{array}$ & $\begin{array}{r}\Delta V \\
(\mathrm{mmag})\end{array}$ & \multicolumn{1}{c}{$S / N$} \\
\hline$f_{0}=25.5769$ & 146.8 & 54.4 \\
$2 f_{0}$ & 29.8 & 14.9 \\
$3 f_{0}$ & 4.4 & 3.6 \\
$f_{1}=31.5927$ & 11.5 & 5.7 \\
$f_{2}=25.2735$ & 33.8 & 12.5 \\
$f_{3}=50.8521$ & 14.1 & 7.0 \\
$\left(\sim f_{0}+f_{2}\right)$ & & \\
$f_{5}=25.9377$ & 21.3 & 7.9 \\
$f_{7}=25.6441$ & 11.8 & 4.4 \\
$f_{8}=51.5088$ & 11.4 & 5.7 \\
$\left(\sim f_{0}+f_{5}\right)$ & & \\
\hline
\end{tabular}

Table 7 summarizes the frequencies detected close to the main pulsation $f_{0}$ from different sets. We confirm the independent modes at $\sim 25.24 \mathrm{~cd}^{-1}, \sim 25.92 \mathrm{~cd}^{-1}$ and $\sim 25.65 \mathrm{~cd}^{-1}$, values very close to those already found by Zhou et al. (1999). From our data sets, the best is that collected in 2003 at Xinglong Station, although only 3 nights were devoted to observations in the Johnson $V$ filter. One secondary peak was detected at $24.220 \mathrm{~cd}^{-1}$ with low amplitude $(7.5 \mathrm{mmag})$ and $S / N$ ratio $(S / N=3.0)$. Moreover, when the 2003 data set collected at Château-Renard Observatory is investigated, a secondary significant peak is found at $23.904 \mathrm{~cd}^{-1}$ with $S / N=4.3$.

As shown in Table 7, changes in amplitude are evident for the secondary peaks detected in the region of $25 \mathrm{~cd}^{-1}$. Moreover, most of them present quite low amplitudes and low $S / N$ ratio, which means that they can appear at the extreme limit of, or beyond, reliability.

\subsection{Discussion}

\subsubsection{Amplitude variations of the main frequency and stability in the light curves}

In Sect. 4.1.1, we have seen that the amplitude of the main frequency $f_{0}$ seems to be stable (Table 3 ), within our errors with the light curves appearing rather regular "on the average" (e.g. most of Zhou et al.'s 1999 observations). However, a puzzling problem in the pulsation analysis comes from the fact that, from time to time, amplitude variations can appear in the form of progressive decreases or increases on the height of the maximum (e.g. at HJD 2449750, Hintz et al. 1997, Fig. 2; or at HJD 2452925 , Fig. 1 of this paper), as well as on the minima (e.g. at HJD 2449748.7, $d=6799.0$ in Fig. 6 in
Table 6. Results of Fourier analysis applied to the 1999 data set of Zhou et al. (2001). The sigma of the residuals is $13.7 \mathrm{mmag}$.

\begin{tabular}{lrr}
\hline \hline $\begin{array}{l}\text { Frequency } \\
\left(\mathrm{cd}^{-1}\right)\end{array}$ & $\begin{array}{r}\Delta V \\
(\mathrm{mmag})\end{array}$ & \multicolumn{1}{c}{$S / N$} \\
\hline & \pm 0.5 & \\
$f_{0}=25.5769$ & 145.7 & 132.5 \\
$2 f_{0}$ & 30.4 & 30.4 \\
$3 f_{0}$ & 4.4 & 6.3 \\
$f_{1}=31.6759$ & 7.0 & 7.0 \\
$f_{2}=25.2469$ & 7.6 & 6.9 \\
$f_{3}=50.8312$ & 4.1 & 4.6 \\
$\left(\sim f_{0}+f_{2}\right)$ & & \\
$f_{4}=32.5456$ & 5.6 & 5.6 \\
$f_{5}=25.9122$ & 5.1 & 4.6 \\
$f_{6}=33.1129$ & 4.8 & 4.8 \\
$f_{7}=25.6653$ & 4.7 & 4.3 \\
\hline
\end{tabular}

Table 7. Frequencies detected close to the main pulsation $f_{0}$. Sources as in Table 3.

\begin{tabular}{cccrcc}
\hline \hline Year & $\begin{array}{c}\text { Frequency } \\
\left(\mathrm{cd}^{-1}\right)\end{array}$ & $\begin{array}{c}\Delta V \\
(\mathrm{mmag})\end{array}$ & $S / N$ & Source & Notes \\
\hline 1995 & 25.2213 & $9.5( \pm 1.3)$ & 4.8 & 1 & \\
& & & & & \\
1996 & 25.2735 & $33.8( \pm 1.1)$ & 12.5 & 3 & \\
1996 & 25.9377 & $21.3( \pm 1.1)$ & 7.9 & 3 & \\
1996 & 25.6441 & $11.8( \pm 1.1)$ & 4.4 & 3 & \\
& & & & \\
1999 & 25.2469 & $7.6( \pm 0.5)$ & 6.9 & 4 & \\
1999 & 25.9122 & $5.1 \pm 0.5)$ & 4.6 & 4 & \\
1999 & 25.6653 & $4.7( \pm 0.5)$ & 4.3 & 4 & \\
& & & & & \\
2000 & 25.239 & $7.9( \pm 1.3)$ & 5.1 & 5 & \\
& & & & & \\
2001 & 24.633 & $9.4( \pm 1.3)$ & 6.2 & 6 & $\mathrm{a}$ \\
& 25.928 & $9.9( \pm 2.6)$ & 2.6 & 5 & \\
2003 & 23.904 & $17.3( \pm 1.0)$ & 4.3 & 2 & $\mathrm{~b}$ \\
& 24.220 & $7.5( \pm 1.0)$ & 3.0 & 7 & $\mathrm{c}$ \\
\hline
\end{tabular}

Notes: a) Possible alias of $\sim 25.65 \mathrm{~cd}^{-1}$; b) Possible alias of $\sim 25.92 \mathrm{~cd}^{-1}$; c) Possible alias of $\sim 25.24 \mathrm{~cd}^{-1}$.

Zhou et al. 1999). In our runs, we observed amplitude variations of $\sim 25-35 \%$ on very close successive cycles (see Sect. 3 ), and an extreme case of such sudden amplitude variation from cycle to cycle is found in Blake et al. (2000), with amplitude variations reaching $\sim 40 \%$ (increase/decrease) at half cycle distances (their Fig. 3). Blake et al. (2000) emphasized that such amplitude modulations seem to be more pronounced in their $I$ bandpass filter.

The existence of multiperiodicity can cause modulations of the light curve. However, such rapid and large amplitude variations, as those mentioned above, are difficult to understand in terms of simple close additional frequencies (the energy available in the pulsation should vary by a factor $\sim 3$ according to Blake et al.'s (2000) observations). These abrupt variations can in no way be related to the frequencies determined here, nor can they be the product of a beat/superposition of multiple frequencies at their detected amplitudes. Thus, we suggest that nonpulsation phenomena are superimposed on the multifrequency solution. These perturbations could be due to strong stellar activity appearing at the level of the star's photosphere, bound to the inner layers of the star, or brought by the rather violent pulsation, into the star's atmosphere. 


\subsubsection{Double-mode star and nature of the pulsation modes}

According to Sect. 4.1.2, the existence of a secondary frequency $f_{1}$ in the $31-32 \mathrm{~cd}^{-1}$ range is confirmed, with a mostlikely value of $\sim 31.6 \mathrm{~cd}^{-1}$, though another independent frequency at $\sim 32.6 \mathrm{~cd}^{-1}$ could also exist. However, the question of these being independent frequencies remains open as long as we do not have multi-longitude coordinated data.

Although Nemec et al. (1994) have initially assigned a first overtone status to the principal frequency of BL Cam, one can argue that it corresponds to the fundamental mode $f_{0}$ of radial pulsation, on the following bases: (i) the atmospheric parameters derived, e.g. in Zhou et al. (1999), lead to a pulsation constant $Q$ of about 0.031; (ii) McNamara $(1995,1997)$ pointed out that the pulsating stars of this type with $V$ amplitudes larger than $\sim 0.25$ mag and asymmetric light curves are fundamental mode pulsators; (iii) as discussed in (Sect. 3), for $1996 B$ and $R$ data, the $R$ maxima lag the $B$ ones. Taking into account $f_{0}, 2 f_{0}$ and $3 f_{0}$, the Fourier analysis of these data leads to a positive phase-shift of 0.014( \pm 0.003$)$, which suggests a radial nature for the $f_{0}$ mode (Balona \& Evers 1999).

We will now examine the real frequency and nature of $f_{1}$. If the frequency of $f_{1} \sim 32.6 \mathrm{~cd}^{-1}$ is assumed, the ratio $f_{0} / f_{1} \sim$ 0.785 is compatible with a radial first overtone identification for $f_{1}$. Given the extreme low metallicity of BL Cam, this period ratio is in agreement with the relations "period ratio versus metallicity" established for double-mode high-amplitude $\delta$ Sct and SX Phe pulsators (Hintz et al. 1997; Kim et al. 2003). This $f_{1}$ value is also consistent with Petersen diagrams for period ratio versus period (Petersen \& Christensen-Dalsgaard 1996; Olech et al. 2005): the theoretically predicted period ratios $\pi_{1} / \pi_{0}$ increase quickly towards shorter periods, with differences for stars in the $0.04-0.125$ range arrising mainly from differences in metallicity. BL Cam should also fit the Petersen diagrams shown in Poretti et al. (2005) where it can be compared with a sample of Population I and II double-mode high-amplitude pulsators. It can be seen in these diagrams that the main differences in the period ratios come from differences in mass for the longer periods $(P>0$ d.15).

However, if we assign $f_{1} \sim 31.6 \mathrm{~cd}^{-1}$ and thus a ratio of $f_{0} / f_{1} \sim 0.810$, our actual period ratio is too high at any metallicity for Petersen's $\pi_{1} / \pi_{0}$ diagrams, and is too low at low metallicities for $\pi_{2} / \pi_{1}$ diagrams (Olech et al. 2005). The 0.810 value could only be identified with that derived from first and second overtones for radial pulsation at solar abundances (Stellingwerf 1979). As this is unrealistic for BL Cam, the nonradial interpretation is best suited for $f_{1}$.

Furthermore, if we consider the four best data sets in Table 3 , i.e. those of Hintz et al. (1997), Zhou et al. (1999), Zhou et al. (2001) and the present work (2003 data set collected at Xinglong Station), the corresponding amplitudes for $f_{0}$ are $146.5,146.8$, 145.7 and $146.9 \mathrm{mmag}$, and those for $f_{1}$ are 9.0, 11.5, 7.0 and $9.6 \mathrm{mmag}$. It suggests that, although the amplitude of $f_{0}$ seems to be very stable, there could be some real variation in the amplitude of $f_{1}$. If this is true, it could be an indication of nonradial pulsation for this mode, although one has to examine very carefully the corresponding amplitude $S / N$ ratio at the level of millimagnitudes. Nevertheless, it might also be that the amplitudes listed for $f_{1}$ correspond to a mixture of modes at $\sim 31.6$ and $\sim 32.6 \mathrm{~cd}^{-1}$ with both peaks being real pulsation modes. Hence, new and precise photometric observations should be crucial to discern if both peaks are real. Moreover, multicolour observations should also be very useful to discriminate their radial or nonradial nature.
Table 8. Frequencies detected close to $f_{0}$. Only peaks with $S / N \geq 4.0$ are listed.

\begin{tabular}{cccc}
\hline \hline Year & $\begin{array}{c}\sim 25.25 \\
\left(\mathrm{~cd}^{-1}\right)\end{array}$ & $\begin{array}{c}\sim 25.65 \\
\left(\mathrm{~cd}^{-1}\right)\end{array}$ & $\begin{array}{c}\sim 25.92 \\
\left(\mathrm{~cd}^{-1}\right)\end{array}$ \\
\hline 1995 & 25.2213 & & \\
1996 & 25.2735 & 25.6441 & 25.9377 \\
1999 & 25.2469 & 25.6653 & 25.9122 \\
2000 & 25.239 & & \\
2001 & & $24.633\left(^{*}\right)$ & \\
2003 & & & $23.904\left(^{*}\right)$ \\
$\Delta V($ mmag $)$ & $\sim 8$ to 34 & $\sim 5$ to 12 & $\sim 5$ to 21 \\
\hline
\end{tabular}

(*) Possible alias (see Notes a), b) in Table 7).

\subsubsection{Multiperiodicity and microvariability in the $25 \mathrm{~cd}^{-1}$ region}

The frequencies detected near $f_{0}$ (Table 7) seem to cluster in three groups around 25.25( \pm 0.03$), 25.65( \pm 0.02)$, and $25.92( \pm 0.02) \mathrm{cd}^{-1}$. Their occurrence was found as significant in 4, 3 and 3 times, respectively, if we include the two detected aliases at a 1 or $2 \mathrm{~cd}^{-1}$ "distance" (Table 8). Some other frequency peaks have been detected at $24.22 \mathrm{~cd}^{-1}$ in 2003 and at $25.93 \mathrm{~cd}^{-1}$ in 2001 , although both with $S / N$ values under 4.0.

The amplitudes of peaks near $f_{0}$ are relatively small and undergo large variations inside each of the three groups. The two most significant ones (i.e. $\sim 25.25 \mathrm{~cd}^{-1}$ and $\sim 25.92 \mathrm{~cd}^{-1}$ groups) are nearly symmetric on each side of the main frequency $f_{0}=25.58 \mathrm{~cd}^{-1}$. We cannot distinguish whether it is a physical splitting of this frequency $f_{0}$ (if the star rotates in a few days) or if it is a pure mathematical artefact, but it is likely not a coincidence. A small distorsion of the light curve or a simple modulation of the main frequency and/or of its amplitude during short observation sessions could create, in the Fourier analysis, some spurious frequencies quite close to the principal peak. Considering that it has been reported by several authors using various data sets with differing alias issues, we believe they are most likely intrinsic pulsations within the star.

According to our analysis, performed with old and new data sets in a homogeneous framework, multiperiodicity probably exists around $25.25,25.65$ and $25.92 \mathrm{~cd}^{-1}$. However, these secondary frequencies should be checked and confirmed from longer-duration observations and/or multi-site photometric campaigns.

\section{The O-C diagram}

\subsection{Description}

Figure 3 shows the evolution of the $\mathrm{O}-\mathrm{C}$ values with Heliocentric Julian Dates, calculated for the main frequency $f_{0}$, using 415 available times of light maximum of Berg \& Duthie (1977), Blake et al. (2000), Hintz et al. (1997), Kim et al. (2003), McNamara \& Feltz (1978), Rodríguez et al. (1990), Van Cauteren \& Wils (2000), Wolf et al. (2002) and Zhou et al. (1999, 2001), and our new maxima (Table 2). In this diagram we converted the light minima given by Van Cauteren \& Wils (2000) to maxima by adding 0.58 of a period, according to the phase lag determined above (Sect. 3 ). Due to the very large discrepancy with the rest of their data, five light maxima of Zhou et al. (1999) were not taken into account, at HJD 2448297.3617 and .3958, 2448298.1000 and .1397, 2448299.0367. 


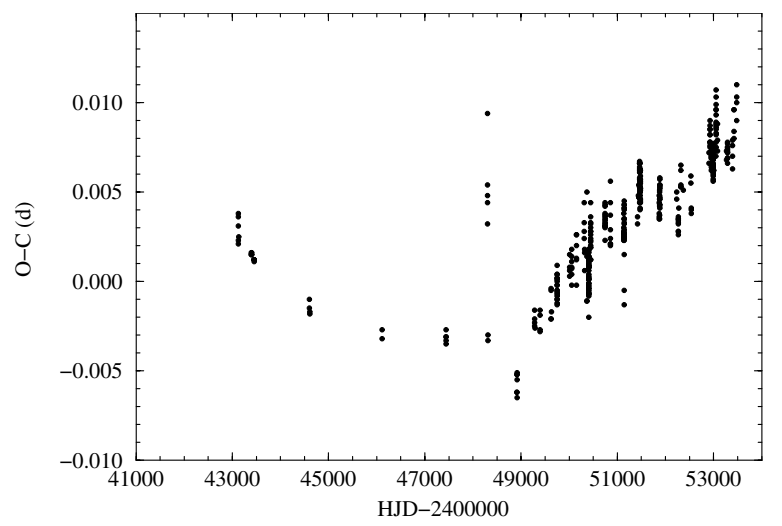

Fig. 3. O-C diagram for BL Cam using the ephemeris of Zhou et al. (1999, Eq. (2)).

The $\mathrm{O}-\mathrm{C}$ values were calculated using the linear ephemeris given by Zhou et al. (1999, Eq. (2)): $\operatorname{HJDmax}(d)=$ $2443125.8015+0.03909785 \times E$, where $E$ is the cycle number. The resulting $\mathrm{O}-\mathrm{C}$ diagram (Fig. 3 ) shows three main regions:

(i) A clear parabolic zone (HJD 2443125 to 2450852 or 21 years) displaying a steady period increase $(0.02 \mathrm{~s}$ in the last 20 years; see Hintz et al. (1997) corrected by Breger \& Pamyatnykh 1998). We confirm this $0.020( \pm 0.004) \mathrm{s} / 20$ years (i.e. $1.1( \pm 0.2) \times 10^{-7} \mathrm{sc}^{-1}$ or $\left.\mathrm{d} P / P \mathrm{~d} t=304( \pm 6) \times 10^{-9} \mathrm{yr}^{-1}\right)$ average period increase.

(ii) A more confused zone (HJD 2451416 to 2452530 , i.e. 3 years) that was first interpreted as a period decrease caused by binarity (reversed trend, Kim et al. 2003). More recent observations (Wolf et al. 2002) showed that this was indeed an almost horizontal feature in the $\mathrm{O}-\mathrm{C}$ diagram, with a rather large vertical dispersion.

(iii) The third part of the diagram (our 2003 to 2005 observations) shows that BL Cam has resumed its period increase (if we only consider the last 2.6 years, i.e about HJD 2452520 to 2453478 , the mean period should have increased at least by $1.29 \times 10^{-7} \mathrm{dc}^{-1}$ or $\sim 0.0003 \%$ per cycle).

The variable shape/amplitude of the star light variations could account for a large part of the typical $\sim 0.002$ vertical dispersion in the $\mathrm{O}-\mathrm{C}$ diagrams. As already pointed out by Hintz et al. (1997), the combination of the two $f_{0}$ and $f_{1}$ frequencies with their respective amplitudes can explain part of the intrinsic dispersion of the light maxima plotted in the $\mathrm{O}-\mathrm{C}$ diagrams, around the mean value given by the $f_{0}$ frequency (e.g., in our 2003 data, it leads to a \pm 0.0005 to 0.0006 dispersion in the $\mathrm{O}-\mathrm{C}$ diagram plotted for frequency $f_{0}$ ).

\subsection{Interpretation as a light travel time (LTT) effect}

The last 4500 days of the $\mathrm{O}-\mathrm{C}$ diagram display significant deviations from the mean parabola of a linear period increase. These deviations could be either abrupt changes (revealing adjustments of the principal pulsation period) as suggested by Koen (2000), or cyclic variations due to light travel time effect in a binary. Indeed, when the parabolic fit is removed from the $\mathrm{O}-\mathrm{C}$ diagram an almost cyclic variation remains.

An explanation could be small transitory readjustments of the internal structure of the star (around the mean secular trend shown by the parabolic $\mathrm{O}-\mathrm{C}$ behaviour), generating small discontinuous "jumps" between stable but transient periods. Given

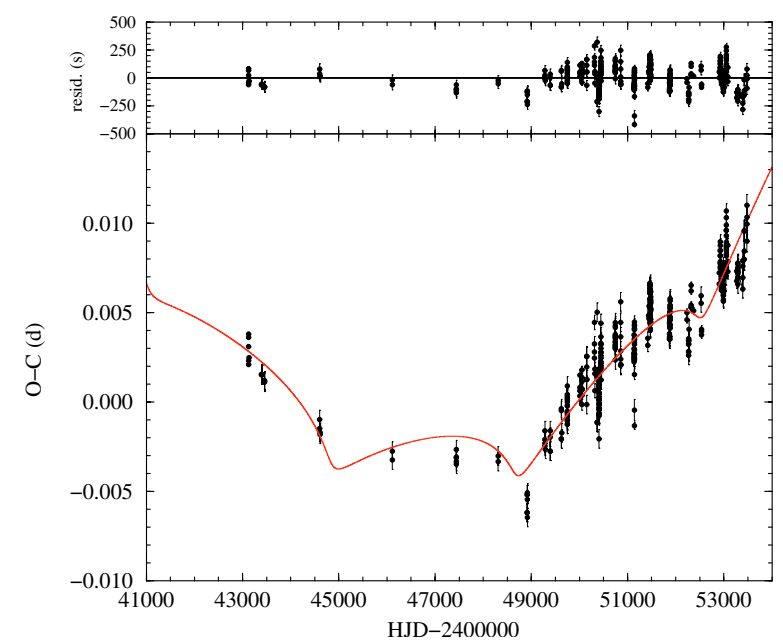

Fig. 4. O-C diagram for BL Cam. Bottom: O-C values (dots) together with the synthetic curve (solid line) using the parameters of the binary system given in Table 9. Top: residuals from the fitting.

Table 9. Orbital elements of the possible BL Cam binary system.

\begin{tabular}{ll}
\hline \hline LTT semi-amplitude (s) & $148( \pm 12)$ \\
Eccentricity & $0.7( \pm 0.2)$ \\
Argument of periastron $(\mathrm{deg})$ & $236( \pm 15)$ \\
Orbital period $(\mathrm{yr})$ & $10.5( \pm 0.2)$ \\
Periastron passage & HJD 2 448 673( \pm 138$)$ \\
\hline
\end{tabular}

the dispersion in the observed $\mathrm{O}-\mathrm{C}$ diagram, this could mimic an oscillation. However, a hybrid adjustment using a parabola and two straight-line segments respectively for the first and last two parts of the $\mathrm{O}-\mathrm{C}$ diagram does not give a satisfactory fit: an oscillatory trend remains. As such, discontinuous period jumps are not completely excluded, although we do not favour this hypothesis.

Accordingly, we have carried out a binary analysis of the $\mathrm{O}-\mathrm{C}$ diagram. The expressions that describe the light-travel time (LTT) effect as a function of the orbital properties were first provided by Irwin (1952). In our work, we used Eq. (1) of Ribas et al. (2002), which is similar to Eq. (2) of Irwin (1952). The $\mathrm{O}-\mathrm{C}$ timings obtained after HJD 2449000 were selected to cover about one cycle of the possible secondary body. With the full set of timings (and their corresponding uncertainties) given by the different authors, fits were run with given weights proportional to $1 /\left(\operatorname{sigma}^{2}\right)$. When no uncertainty was available, we adopted a median uncertainty value of \pm 0 d0005.

The following parameters were considered in our fits: semiamplitude, eccentricity, argument of periastron, orbital period, date of periastron passage. Solutions with free eccentricity were found to be unstable. This is because the fit quality is degenerate with respect to this parameter and the fits can attain arbitrarily large values. So fits were run by fixing eccentricity to values between 0 and 1 at regular steps. The fits clearly prefer highly eccentric values, greater than 0.5 . When the eccentricity changes from 0.5 to 0.7 and then to 0.9 , the improvement is hardly perceptible. As such, a value of $0.7( \pm 0.2)$ was adopted. We obtained a tentative period of $10.5( \pm 0.2) \mathrm{yr}$ with a semiamplitude of $148( \pm 12) \mathrm{s}$ in O-C. The set of orbital elements of the binary system are presented in Table 9.

Figure 4 (bottom) shows the $\mathrm{O}-\mathrm{C}$ diagram fitted with the parameters of Table 9 . The root mean square residual (top of Fig. 4) is \pm 0.001 , and residuals do not show any systematic trends. 


\subsection{Discussion}

Based on analysis of the $\mathrm{O}-\mathrm{C}$ residuals, some high-amplitude pulsators in the Lower Instability Strip have been reported as or suspected to be members of binary systems (Rodríguez 2003), BL Cam being one of the candidates.

Previous authors have estimated the increasing rate of the main period of BL Cam with a parabolic fit. Hintz et al. (1997) and Zhou et al. (1999) found respectively $\mathrm{d} P / P \mathrm{~d} t$ values of $290 \times$ $10^{-9} \mathrm{yr}^{-1}$ and $171 \times 10^{-9} \mathrm{yr}^{-1}$. In the same way, with all available and new $\mathrm{O}-\mathrm{C}$ data, we find $188( \pm 8) \times 10^{-9} \mathrm{yr}^{-1}$. However, in the case of the binarity hypothesis, the $\mathrm{O}-\mathrm{C}$ diagram can be better described by a secular increase of the period at a rate of $\mathrm{d} P / P \mathrm{~d} t=117( \pm 3) \times 10^{-9} \mathrm{yr}^{-1}$ (i.e. $\left.1.08( \pm 0.03) \times 10^{-6} \mathrm{~s} \mathrm{~d}^{-1}\right)$, in addition to the $10.5( \pm 0.2) \mathrm{yr}$ orbital period of the system.

We can draw up a tentative representation of the system of BL Cam. Using Kepler's Third Law, and adopting a mass of $\sim 1 M_{\odot}$ for BL Cam (McNamara 1997), Table 9 indicates a range in mass of 0.1 to $0.5 M_{\odot}$ (with a minimum of $\sim 0.1 M_{\odot}$ ) for the secondary body with a semi-major axis of 5-6 AU. As we did not find any eclipse in the light curves, the inclination of this possible high eccentricity binary system cannot be determined.

However, some observational evidence is opposed to the binary hypothesis. Though there is no obvious systematic trend in the scatter of the residuals in Fig. 4 (top panel), we note that the latest O-C values (HJD 2453264 to 2453478 ) have residuals larger than expected from the LTT effect model based on our previous values (Fig. 4). As already mentioned in Sect. 5.1, there is a large dispersion ( $\sim 002)$ in the $\mathrm{O}-\mathrm{C}$ diagram, so the times of last light maxima are not, up to now, sufficient to discard the binarity hypothesis. Indeed, very recent O-C data (Simonetti, private communication) confirm the plausibility of the binary hypothesis. Finally, as the interpretation of the $\mathrm{O}-\mathrm{C}$ diagram requires observations over a long time base, new observations during several years are necessary to definitively conclude on the existence of a binary perturbation.

This should be of crucial importance in our understanding of the processes taking place in BL Cam and stars of similar evolutionary status. As discussed in previous reviews (Breger \& Pamyatnykh 1998; Rodríguez 2003), the large and positive rate of period change determined in BL Cam is not predicted by the evolutionary tracks. The observed rate is much larger than predicted by the models. On the one hand, the binary hypothesis could be a partial explanation of this discrepancy. Binarity could also be the cause of similar disagreements found for other high-amplitude $\delta$ Sct and SX Phe variables (Rodríguez \& Breger 2001). On the other hand, the existence of jumps in the $\mathrm{O}-\mathrm{C}$ diagram of BL Cam should not be completely ruled out. Possible sudden jumps could occur in a number of these variables, although they are commonly shown as an alternative to parabolic fits and the choice between the two options is not an easy task.

Our observed period variation in BL Cam is also positive, which is consistent with all the previous determinations. However, such period variation is opposite to that observed in the rest of the high-amplitude SX Phe pulsators with reliable determinations, with the possible exception of XX Cyg (Zhou et al. 2002; and Blake et al. 2003 find $\mathrm{d} P / P \mathrm{~d} t \sim 10 \times 10^{-9} \mathrm{yr}^{-1}$ for this star). This makes BL Cam a very interesting object: the precise determination of its $\mathrm{O}-\mathrm{C}$ behaviour will give us insights on what part of the period change rate can be explained by standard stellar evolution and what part explained by non-evolutionary effects. It could also give us some insight on the role of the nonevolutionary effects in the $\mathrm{O}-\mathrm{C}$ diagrams.

\section{Conclusions}

A comprehensive study of the high-amplitude SX Phe-type pulsator BL Cam is presented. An investigation of the pulsation content of this variable has been performed, using both previously published data and our new observations obtained in 1996, and during the years 2003 to 2005 .

Our frequency analysis confirms the existence of multiperiodicity in this star, as pointed out by previous authors. Independent peaks have been significantly detected in the region close to the main frequency $f_{0}=25.5769 \mathrm{~cd}^{-1}$; in particular at around 25.25, 25.65 and $25.92 \mathrm{~cd}^{-1}$, and which also show signs of amplitude variability. Nevertheless, the main frequency does not exhibit significant amplitude changes and probably corresponds to the fundamental mode of radial pulsation. We also confirm the existence of at least one frequency in the of region $31-32 \mathrm{~cd}^{-1}$. Our results suggest the existence of two independent frequencies in this region at about 31.6 and $32.6 \mathrm{~cd}^{-1}$, but the frequency at $31.64( \pm 0.05) \mathrm{cd}^{-1}$ is the most reliably detected. Sudden amplitude variations in the light curves, within a very few pulsation cycles, suggest that strong stellar activity can occasionally occur and is superimposed upon the pulsation.

These topics make BL Cam a very good and unique target among the field high-amplitude SX Phe-type variables for further studies from an asteroseismological point of view in order to constraint its pulsation parameters. Furthermore, multicolour photometric observations, in at least two filters, will be very important to prove the radial nature of $f_{0}$ and discern the true nature of the intrinsic peaks in the region $31-32 \mathrm{~cd}^{-1}$. If confirmed, the radial double-mode nature of this star would be a significant find as it would add constraints to the period-luminosity and/or period-metallicity relations for high-amplitude pulsators in the Lower Instability Strip because of its extreme short period and low metallicity. Improved precision in the period ratios of BL Cam will also improve the Petersen diagrams.

We also studied the behaviour of the main period making use of the classical $\mathrm{O}-\mathrm{C}$ method. The new $\mathrm{O}-\mathrm{C}$ diagram, completed by our 2003 to 2005 measurements, suggests that a sinusoidal behaviour is superimposed to the classical parabolic one since the first half of the 1990's, when the star has been more frequently observed. Although we do not definitely discard discontinuous variations of the main pulsation period, we have shown that the observed variations of the period can be described by two terms: (i) a secular increase of the main period at a rate of $\mathrm{d} P / P \mathrm{~d} t=117( \pm 3) \times 10^{-9} \mathrm{yr}^{-1}$, and (ii) a perturbation from a companion star in a rather eccentric orbit with a period of $10.5( \pm 0.2)$ yr causing a light time semi-amplitude of $148( \pm 12) \mathrm{s}$. To gain insights on the true behaviour on the main period in the $\mathrm{O}-\mathrm{C}$ diagram, it will be of great interest to locate this variable in the observational framework among the highamplitude pulsators in the Lower Instability Strip. This would place direct (and likely opposing) constraints on stellar evolution models, and could provide insight on the possible secondary effects taking place at this stage of stellar evolution (see e.g., Rodríguez et al. 1995; Breger \& Pamyatnykh 1998).

BL Cam obviously deserves continued photometric monitoring in the coming years. Remaining observational issues are to confirm our detected frequency content, pulsation behaviour and checking our $\mathrm{O}-\mathrm{C}$ binary hypothesis. To shed some light on these issues, the organization of a coordinated multisite photometric campaign with observations from several observatories at different longitudes would be of a great importance. We are already working in this direction for the near future. 
Acknowledgements. We would like to give our most sincere thanks to P. Van Cauteren, E. Hintz, C. Kim, M. Wolf, P. Delaney, R. Robb and J. Simonetti for making available different data sets used in this work. Also thanks to M. Newberry (Axiom Research, Inc.), P. Lampens (Koninklijke Sterreanwacht van België), E. Michel (observatoire de ParisMeudon), C. Sterken (Vrije Universiteit Brussel) and R. Pellock (AAVSO). E.R. thank for the support by the Junta de Andalucía and the Dirección General de Investigación (DGI) under project AYA2003-04651. J.M. especially acknowledges R. Behrend (observatoire de Genève) who provided the Courbrot software. S.F. is grateful to M. Fauvaud, D. Pommier, J. Vandenbroere, A. Bruno, C. Fournou, Y. Pothier, D. and F. Béziat for their help and technical assistance during observing runs. This research has made use of both the Simbad database, operated at CDS, Strasbourg, France, and the Astrophysics Data System, provided by NASA, USA. MDR and A.-Y. Zhou were supported by the National Science Foundation grant AST007480.

\section{References}

Balona, L. A., \& Evers, E. A. 1999, MNRAS, 302, 349

Berg, R. A., \& Duthie, J. G. 1977, AJ, 215, L25

Blake, R. M., Khosravani, H., \& Delaney, P. A. 2000, JRASC, 94, 124

Blake, R. M., Delaney, P. A., Khosravani, H., Tome, J., \& Lightman, M. 2003, PASP, 115, 212

Breger, M., \& Pamyatnykh, A. A. 1998, A\&A, 332, 958

Breger, M., Stich, J., Garrido, R., et al. 1993, A\&A, 271, 482

Breger, M., Handler, G., Serkowitsch, E., et al. 1996, A\&A, 309, 197

Breger, M., Handler, G., Garrido, R., et al. 1999, A\&A, 349, 225

Eggen, O. J., \& Iben, I. Jr. 1989, AJ, 97, 431

Garrido, R., \& Rodríguez, E. 1996, MNRAS, 281, 696

Giclas, H. L., Burnham, R. Jr., \& Thomas, N. G. 1970, Lowell Obs. Bull., 7, 183

Hintz, E. G., Joner, M. D., McNamara, D. H., Nelson, K. A., \& Moody, J. W. 1997, PASP, 109,15

Høg, E., \& Petersen, J. O. 1997, A\&A, 323, 827

Irwin, J. B. 1952, ApJ, 116, 211

Jeon, Y. B., Lee, M. G., Kim, S. L., \& Lee, H. 2003, AJ, 125, 3165

Jeon, Y. B., Lee, M. G., Kim, S. L. \& Lee, H. 2004, AJ, 128, 287

Kim, C., Jeon, Y. B., \& Kim, S. L. 2003, PASP, 115, 755
Koen, C. 2000, PASPC, 210, 448

Livio, M. 1993, PASPC, 53, 3

Mateo, M. 1993, PASPC, 53, 74

Mazur, B., Krzeminski, W., \& Thompson, I. B. 2003, MNRAS, 340, 1205

McNamara, D. H. 1995, AJ, 109, 1751

McNamara, D. H. 1997, PASP, 109, 1221

McNamara, D. H., \& Feltz, K. A. 1978, PASP, 90, 275

Nemec, J. M., \& Mateo, M. 1990, PASPC, 11, 64

Nemec, J. M., Nemec, A. F. L., \& Lutz, T. E. 1994, AJ, 108, 222

Olech, A., Dziembowski, W. A., Pamyatnykh, A. A., et al. 2005, MNRAS, 363, 40

Petersen, J. O., \& Christensen-Dalsgaard, J. 1996, A\&A, 312, 463

Pócs, M. D., Szeidl, B., \& Viraghalmy, G. 2002, A\&A, 393, 555

Poretti, E., Suárez, J. C., Niarchos, P. G., et al. 2005, A\&A, 440, 1097

Preston, G. W., \& Landolt, A. U. 1998, AJ, 115, 2515

Pych, W., Kaluzny, J., Krzeminski, W., et al. 2001, A\&A, 367, 148

Ribas, I., Arenou, F., \& Guinan, E. F. 2002, AJ, 123, 2033

Rodríguez, E. 1999, PASP, 111, 709

Rodríguez, E. 2003, Recent Res. Devel. Astron. Astroph., 1, 881

Rodríguez, E. 2004, Comm. Asteroseismology, 145, 40

Rodríguez, E., \& Breger, M. 2001, A\&A, 366, 178

Rodríguez, E., \& López-González, M. J. 2000, A\&A, 359, 597

Rodríguez, E., Rolland, A., \& López de Coca, P. 1990, IBVS, 342

Rodríguez, E., López de Coca, P., Costa, V., \& Martín, S. 1995, A\&A, 299, 108

Rodríguez, E., Rolland, A., López-González, M. J., \& Costa, V. 1998, A\&A, 338, 905

Rodríguez, E., Arellano-Ferro, A., Costa, V., López-González, M. J., \& Sareyan, J. P. 2003, A\&A, 407, 1059

Stellingwerf, R. F. 1979, ApJ, 227, 935

Stryker, L. L. 1993, PASP, 105, 1081

Van Cauteren, P., \& Wils, P. 2000, IBVS, 4872

Walraven, T., Walraven, J., \& Balona, L. A. 1992, MNRAS, 254, 59

Wolf, M., Crlikova, M., Basta, M., et al. 2002, IBVS, 5317

Zhou, A. Y., Rodríguez, E., Jiang, S. Y., Rolland, A., \& Costa, V. 1999, MNRAS, 308, 631

Zhou, A. Y., Du, B. T., Zhang, X. B., \& Zhang, R. X. 2001, IBVS, 506

Zhou, A. Y., Jiang, S. Y., Chayan, B. \& Du, B. T. 2002, Ap\&SS, 281, 699

Zhou, A. Y., An, D., Eggen, J. R., et al. 2006, Ap\&SS, submitted 